\title{
Annual prevalence and economic burden of genital warts in Korea: Health Insurance Review and Assessment (HIRA) service data from 2007 to 2015
}

\author{
Y. J. PARK ${ }^{1}$, J. M. KIM ${ }^{2}$, B. R. LEE ${ }^{3}$, T. H. KIM ${ }^{4}$ AND E. G. LEE ${ }^{1}$ \\ ${ }^{1}$ Department of Interdisciplinary Programme in Biomedical Science, Soonchunhyang University Graduate \\ School, Asan, Korea \\ ${ }^{2}$ Department of Urology, Soonchunhyang University College of Medicine, Soonchunhyang Buchoen Hospital, \\ Bucheon, Korea \\ ${ }^{3}$ Department of Biostatistic Consulting, Clinical Trial Centre, Soonchunhyang University Bucheon Hospital, \\ Bucheon, Korea \\ ${ }^{4}$ Department of Obstetrics and Gynecology, Soonchunhyang University College of Medicine, Soonchunhyang \\ Buchoen Hospital, Bucheon, Korea
}

Received 3 August 2017; Final revision 31 October 2017; Accepted 14 November 2017;

first published online 13 December 2017

\section{SUMMARY}

This study evaluated the annual prevalence of anogenital warts (AGW) caused by human papillomavirus (HPV) and analysed the trend in annual per cent changes (APC) by using national claims data from the Health Insurance Review and Assessment of Korea, 2007-2015. We also estimated the socio-economic burden and co-morbidities of AGW. All analyses were performed based on data for primary A63.0, the specific diagnosis code for AGW. The socioeconomic cost of AGW was calculated based on the direct medical cost, direct non-medical cost and indirect cost. The overall AGW prevalence and socio-economic burden has increased during the last 9 years. However, the prevalence of AGW differed significantly by sex. The female prevalence increased until 2012, and decreased thereafter (APC $+3 \cdot 6 \%$ ). It would fall after the introduction of routine HPV vaccination, principally for females, in Korea. The male prevalence increased continuously over time (APC $+11 \cdot 6 \%$ ), especially in those aged $20-49$ years. Referring to the increasing AGW prevalence and its disease burden, active HPV infection control surveillance and prevention in males are worth consideration.

Key words: Big data analysis, genital warts, human papillomavirus vaccine, sexually transmitted disease, urogenital infection.

\section{INTRODUCTION}

Anogenital human papillomavirus (HPV), one of the most common sexually transmitted viral infections, is a double-stranded virus causing several types of warts and cancers in both males and females [1]. About $50 \%$ of sexually active subjects are at risk of acquiring

\footnotetext{
* Author for correspondence: J.-M. Kim, Department of Urology, Soonchunhyang University Bucheon Hospital, 170 Jomaru-ro, Wonmi-gu, Bucheon 14584, Korea.

(Email: urojun@schmc.ac.kr)
}

a urogenital HPV infection that they may pass on to their partners [2]. Among the 210 officially recognised HPV types, low-risk types of HPV (e.g. types 6 and 11) cause $90 \%$ of all cases of anogenital warts (AGW) [3, 4]. HPV infection negatively impacts economic status and quality-of-life, attributable to anxiety, embarrassment, shame and lack of sexual activity and enjoyment [5]. HPV infections and AGW not only reduce sperm vitality in males but also increase the likelihood of transmission to their sexual partner, as 36\% of HPV infections in men remain persistent 24 months 
after their initial detection [6]. They can cause female infertility, trigger extrauterine pregnancies and contribute to early foetal death [7].

The prevalence of HPV infections has increased over the past four decades [8]. Approximately 79 million subjects are currently infected with HPV, and about 14 million subjects aged 15-59 years become newly infected with genital HPV annually in the United States (US). Approximately half of all new infections develop in those aged 15-24 years $[9,10]$. The prevalence of HPV infection is high among Korean males. Among sexually active undergraduates in South Korea, the prevalence of the infection is $38.8 \%$ in females and $10.6 \%$ in males [11]. The prevalences of low- and high-risk HPV infections (e.g. infections caused by HPV types 16 and 18) in males with genital warts are $97 \cdot 0 \%$ and $23.5 \%$, respectively. Of all infected males, $20 \cdot 5 \%$ exhibited both high- and low-risk HPV infections [12].

Gardasil $^{\mathrm{TM}}$ (Merck \& Co., Inc., Whitehouse Station, NJ, US) is a prophylactic quadrivalent vaccine protecting against HPV types 6,11,16 and 18, which can cause AGW and various cancers and pre-cancers [13]. Cervarix $^{\mathrm{TM}}$ (GlaxoSmithKline, Middlesex, UK) is a bivalent vaccine protecting against, e.g. types 16 and 18. Gardasil and Cervarix were approved by the Korean Food and Drug Administration in June 2007 and July 2008, respectively [14]. In 2009, the World Health Organisation (WHO) suggested that prophylactic HPV vaccination should be included in national immunisation programmes (NIPs), and it published guidelines for the three-dose HPV vaccination of females aged 9-13 years [15]. The WHO has changed the previous recommendation of three-dose vaccination to two-dose HPV vaccination in females aged 9-13 years in October 2014 [16]. The Food and Drug Administration and European Medicines Agency approved a nonavalent HPV vaccine, Gardasil 9 (Merck \& Co., Inc., Whitehouse Station, NJ, US), in 2014 and 2015, respectively. It prevents five additional HPV types (HPV 31/33/45/52/58), which are the third to eighth most frequent HPV types after HPV 16 and 18 [17, 18].

Although several national studies have assessed the efficacy of current prophylactic HPV vaccines and trends in the prevalence of AGW, less is known about the epidemiological burden of AGW in males, and no such study has been conducted in South Korea [19-21]. Therefore, we describe the characteristics and prevalence of AGW in both sexes since the introduction of routine HPV vaccination (principally for females) in South Korea. We also estimated the socio-economic burden imposed by AGW, and the co-morbidities thereof, using national 'big data'.

\section{METHODS}

\section{Study subjects}

We analysed the national public organisational database of the HIRA service from 2007 to 2015 to investigate the prevalence and socio-economic burden of AGW in Korea. We collected 9-year claims data for principal diagnosis code A63.0 (AGW or venereal warts according to the International Classification of Disease 10th version (ICD-10)) [22]. Korean medical institutions can use code A63, A63.0 or A63.8 for AGW. A63 and A63.8 are not specific for AGW, whereas A63.0 is specific for AGW. Therefore, all data from individuals with a primary code of A63.0 were included in the analysis, while those with a less specific code (A63 or A63.8) were excluded. This study was approved by the Institutional Review Board (IRB) of Soonchunhyang University Bucheon Hospital (IRB no. SCHBC 2015-03-008).

\section{Data sources}

\section{Health Insurance Review and Assessment data}

Health Insurance Review and Assessment (HIRA) is a national public organisation that reviews medical service fees to ensure that they are appropriate, and that monitors medical quality standards. HIRA maintains an electronic database on inpatients and outpatients treated at all Korean healthcare institutions, healthcare billing and insurance claims. HIRA data have been made publicly available for research purposes. The data can be divided into three categories: general medical details of patients and their diseases, diagnoses and prescription information. In Korea, $97 \%$ of the population is covered by the Korea National Health Insurance Programme (KNHIP). The other 3\% of the population is assisted by the Medical Aid Programme (MAP). Almost all medical information is available from the HIRA database, apart from the details of procedures that are not covered by insurance.

Korean National Health and Nutritional Examination Survey data

Korean National Health and Nutritional Examination Survey (KNHANES) is a national surveillance system evaluating the health-related status of Koreans. 
KNHANES has conducted nationwide cross-sectional study every year since 1998, and provides information on disease epidemiology, trends in socio-economic status, health behaviours, etc. We estimated daily traffic cost to visit clinic using KNHANES IV (2009), V (2010-2012) and VI (2013) data.

\section{Korean Statistical Information Service data}

The Korean Statistical Information Service (KOSIS) database delivers Korean statistics on more than 500 topics that are the responsibilities of 120 agencies. We used total population data, and population numbers by sex and age, to calculate the prevalence of AGW. Employment data and average daily incomes were used to estimate indirect medical costs.

\section{Prevalence of AGW}

All data from individuals with a primary code of A63.0 were included in the prevalence analysis. Cases of recurrence per year were regarded as one case. Any case in which treatment continued into the following year was excluded from calculation of the next year's prevalence. We calculated the annual prevalence of AGW by dividing the number of cases by the residence-registration mid-year population in the corresponding year. The residence-registration mid-year population was obtained from the KOSIS [23]. Prevalences were reported as age-standardised rates (ASRs) using the residence-registration mid-year population in 2011 as a standard.

\section{Socio-economic burden of AGW}

The socio-economic cost of AGW was calculated based on HIRA claim data, KOSIS data and KNHANES data. The socio-economic cost of AGW was classified as direct medical costs, direct non-medical costs and indirect costs; it included expenses for outpatient care or hospitalisation, medicines, prescriptions, traffic expenses and lost income paid by the insurer and patients (including non-covered costs and prescribed pharmaceutical costs). The formulae used for the calculations were reported previously [24, 25].

\section{Direct medical cost}

Direct medical cost was calculated based on HIRA KNHIP and the MAP data paid by the insurer or patient, including deductible payments. It was defined as expenses for outpatient treatment or hospitalisation; medicines and prescriptions; and prescribed pharmaceutical costs. The medical charges for non-insured costs paid by patients were $20.5 \%$ and $2.9 \%$ for visiting clinics/hospitalisation and pharmaceuticals, respectively [26].

Direct medical cost $=\sum_{i} \sum_{j}\left(\frac{O E_{i j}}{1-\alpha}+\frac{E_{i j}}{1-\beta}+\frac{E D_{i j}}{1-\lambda}\right)$

$(i=0,1 \cdots$, age

$j=1,2$, sex

$\alpha=$ proportion of non-insured costs paid by patients for visiting clinics

$\beta=$ proportion of non-insured costs paid by

where $\{$ patients for hospitalisation

$\gamma=$ proportion of non-insured costs paid by

patients for buying medicines

$O E_{i j}=$ amount claimed for visiting clinics

$E_{i j}=$ amount claimed for hospitalisaton

$E D_{i j}=$ amount claimed for buying medicines

\section{Direct non-medical cost}

Direct non-medical cost was defined as transport expenses for visiting clinics or hospitalisation. Based on the 2008 Korea Health Panel Survey, the average roundtrip transport expenses were 1.24 United States Dollar (US\$) and US\$5.60 for visiting clinics and hospitalisation, respectively [27]. The transport expenses were corrected by multiplying the inflation rate (traffic price index) of KOSIS for each year. Because individuals aged $<20$ or more than 64 years were assumed to be with a caregiver when visiting clinics or during hospitalisation, the traffic costs of those age groups were doubled.

\section{Indirect cost}

Indirect cost was defined as lost income caused by decreased productivity due to AGW [28].

Indirect cost $=\sum_{i} \sum_{j}\left(O_{i j} \times \delta+N_{i j}\right) \times R_{i j} \times Y_{i j}$

where $\left\{\begin{array}{l}i=0,1 \cdots, \text { age } \\ j=1,2, \text { sex } \\ O_{i j}=\text { number of visiting clinics } \\ \delta=\text { proportion rate of visiting clinics } \\ \quad \text { versus hospitalisation } \\ N_{i j}=\text { length of hospital stay(days) } \\ R_{i j}=\text { employment rate } \\ Y_{i j}=\text { average daily income }\end{array}\right.$ 

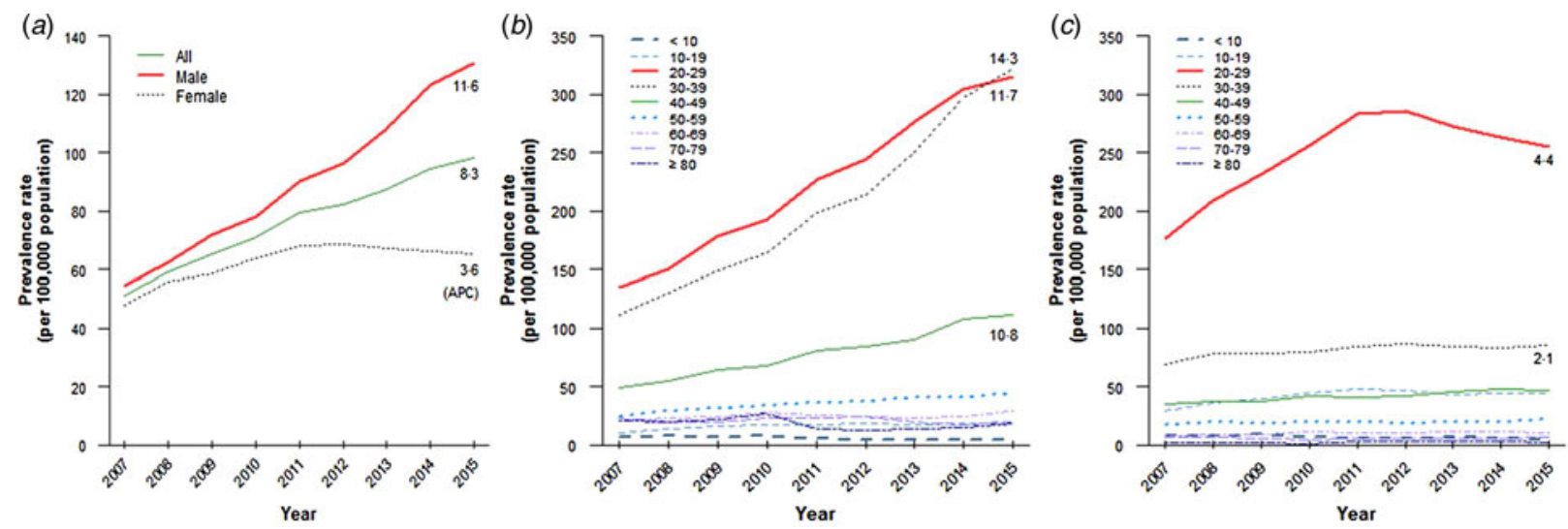

Fig. 1. Tendency of anogenital warts prevalence in Korea, 2007-2015. (a) Prevalence of anogenital warts in the whole population, $(b)$ in male population and $(c)$ in female population.

The proportion rate of visiting clinics $v s$. hospitalisation was set at one-third based on a previous study, which presumed that the cost of treatment of one patient in a ward is equal to that of three patients visiting clinics due to doctor's productivity [29]. The employment rate and average daily income per age and sex were determined from KOSIS data. Patients younger than 20 years or older than 64 years were assumed to have no loss of productivity; however, they were assumed to have caregivers. Therefore, the indirect cost of AGW was estimated by calculating the decreased productivity of the patients' caregivers [25].

\section{Co-morbidities of AGW}

Co-morbidities are co-existing or additional diseases with reference to an initial diagnosis, in this case HPV and AGW. Therefore, diseases unrelated to AGW and HPV infections were excluded when ranking the most frequent co-morbidities of AGW.

\section{Statistical analysis}

The prevalence of AGW according to sex and age was calculated by applying ASRs using the standard population from 2007 to 2015. A two-tailed $P<0.05$ was considered to indicate statistical significance. All statistical analyses were performed using SAS (version 9.3; SAS Institute Inc., Cary, NC, USA) and R (version 3.1.3; The $\mathrm{R}$ Foundation for Statistical Computing, Vienna, Austria). To evaluate the trend in prevalence, the APC in prevalence was calculated using a simple linear regression model: $\mathrm{APC}=(\exp$ $(b)-1) \times 100$, where $b$ is the linear regression coefficient of the natural log-transformed of the ASR for each year; $95 \%$ confidence intervals were calculated from the fitted regression model.

\section{RESULTS}

\section{Prevalence of AGW according to sex}

The overall AGW prevalence in both males and females increased from 2007 to 2015, with an average APC of $+8 \cdot 3 \%$. The number of patients with AGW increased approximately $1 \cdot 8$-fold over the last 9 years (Fig. 1, Table S1).

The AGW prevalence in males increased continuously from 2007 to 2015, and was higher than that in females. The APC in males was $+11 \cdot 6 \%$, and the number of patients increased $>2 \cdot 3$-fold during that period (Fig. 1, Table S1). In contrast, the AGW prevalence in females increased until 2012 and decreased thereafter. The APC in females was 3.2-fold lower than that in males, with APC of $+3 \cdot 6 \%$ (Fig. 1, Table S1).

The male-to-female prevalence rate ratios of AGW for all ages increased steadily from $1 \cdot 1$ (2007) to $2 \cdot 0$ (2015). This indicates that the prevalence in males has increased more than that in females (Table 1).

\section{Prevalence of AGW according to age}

The AGW prevalence was highest in patients aged 20-29 years in both sexes from 2007 to 2015 . The prevalence of AGW was higher in females aged 20-29 years, particularly in 2012, than in the other age groups; however, it decreased thereafter. The prevalence of AGW was higher in males aged 20-29, 30-39 and 40-49 years than in the other age groups, and has increased during the last 9 years. Interestingly, the gap between those aged 20-29 and 30-39 years decreased and reversed 
Table 1. Male-to-female prevalence ratio of anogenital warts in Korea, 2007-2015

\begin{tabular}{|c|c|c|c|c|c|c|c|c|c|c|c|c|}
\hline \multirow[b]{2}{*}{ Ages (years) } & \multicolumn{9}{|l|}{ PRR } & \multirow[b]{2}{*}{ Slope } & \multirow[b]{2}{*}{$(95 \% \mathrm{CI})$} & \multirow[b]{2}{*}{$P$} \\
\hline & 2007 & 2008 & 2009 & 2010 & 2011 & 2012 & 2013 & 2014 & 2015 & & & \\
\hline All & $1 \cdot 1$ & $1 \cdot 1$ & $1 \cdot 2$ & $1 \cdot 2$ & $1 \cdot 3$ & $1 \cdot 4$ & $1 \cdot 6$ & 1.9 & $2 \cdot 0$ & $0 \cdot 12$ & $(0 \cdot 08,0 \cdot 15)$ & $<0.001$ \\
\hline$<10$ & $0 \cdot 9$ & $1 \cdot 0$ & $0 \cdot 8$ & $1 \cdot 2$ & $1 \cdot 0$ & 0.9 & $0 \cdot 8$ & $0 \cdot 9$ & $1 \cdot 0$ & $0 \cdot 00$ & $(-0 \cdot 04,0 \cdot 04)$ & 0.959 \\
\hline $10-19$ & $0 \cdot 4$ & $0 \cdot 4$ & $0 \cdot 4$ & $0 \cdot 4$ & $0 \cdot 4$ & $0 \cdot 4$ & $0 \cdot 4$ & $0 \cdot 4$ & $0 \cdot 4$ & $0 \cdot 01$ & $(0 \cdot 00,0 \cdot 01)$ & 0.036 \\
\hline $20-29$ & $0 \cdot 8$ & $0 \cdot 7$ & $0 \cdot 8$ & $0 \cdot 8$ & $0 \cdot 8$ & $0 \cdot 9$ & $1 \cdot 0$ & $1 \cdot 2$ & $1 \cdot 2$ & $0 \cdot 06$ & $(0 \cdot 04,0 \cdot 09)$ & $0 \cdot 001$ \\
\hline $30-39$ & $1 \cdot 6$ & $1 \cdot 6$ & 1.9 & $2 \cdot 1$ & $2 \cdot 3$ & $2 \cdot 5$ & $3 \cdot 0$ & $3 \cdot 6$ & $3 \cdot 8$ & $0 \cdot 28$ & $(0 \cdot 22,0 \cdot 35)$ & $<0.001$ \\
\hline $40-49$ & $1 \cdot 4$ & $1 \cdot 5$ & $1 \cdot 7$ & $1 \cdot 6$ & $2 \cdot 0$ & $2 \cdot 0$ & $2 \cdot 0$ & $2 \cdot 2$ & $2 \cdot 4$ & $0 \cdot 12$ & $(0 \cdot 09,0 \cdot 14)$ & $<0.001$ \\
\hline $50-59$ & $1 \cdot 4$ & $1 \cdot 4$ & $1 \cdot 7$ & $1 \cdot 7$ & $1 \cdot 8$ & $2 \cdot 1$ & $2 \cdot 0$ & $2 \cdot 1$ & $2 \cdot 1$ & $0 \cdot 09$ & $(0 \cdot 06,0 \cdot 12)$ & $<0.001$ \\
\hline $60-69$ & $2 \cdot 5$ & $2 \cdot 5$ & $2 \cdot 7$ & $2 \cdot 5$ & $2 \cdot 5$ & $2 \cdot 4$ & $2 \cdot 1$ & $2 \cdot 2$ & $3 \cdot 0$ & $-0 \cdot 01$ & $(-0.09,0.08)$ & $0 \cdot 882$ \\
\hline $70-79$ & $3 \cdot 8$ & $2 \cdot 9$ & $3 \cdot 5$ & $4 \cdot 8$ & $4 \cdot 3$ & $4 \cdot 9$ & $3 \cdot 9$ & $3 \cdot 1$ & $3 \cdot 0$ & -0.03 & $(-0 \cdot 28,0 \cdot 22)$ & $0 \cdot 78$ \\
\hline$\geqslant 80$ & $9 \cdot 5$ & $8 \cdot 5$ & $11 \cdot 9$ & $42 \cdot 6$ & $4 \cdot 2$ & $3 \cdot 7$ & $5 \cdot 5$ & $5 \cdot 4$ & $9 \cdot 4$ & $-1 \cdot 02$ & $(-4 \cdot 86,2 \cdot 82)$ & $0 \cdot 549$ \\
\hline
\end{tabular}

PRR, prevalence rate ratio; CI, confidence interval.

from 2015. This indicates that AGW are more prevalent in males aged 30-39 years in Korea. The prevalence of AGW in males had two peaks, at 20-29 and 30-39 years of age, while there was a single peak in females aged 20-29 years (Figs 1 and 2, Table S1).

The APC was highest in patients aged 30-39 $(+10 \cdot 7 \%)$ years, followed by those aged $40-49$ $(+8.4 \%)$ years in both sexes. The APC in those aged $40-49(+10 \cdot 8 \%)$ and $50-59(+7 \cdot 2 \%)$ years was higher than that in those aged $10-19(+6 \cdot 0 \%)$ in males, despite the expectation that middle-aged persons are less sexually active than teenagers are (Fig. 2, Table S1).

\section{Economic burden of AGW in South Korea ${ }^{1}$}

\section{Total cost for the treatment of $A G W$}

The total socio-economic cost increased 2.6-fold from $\$ 3901209$ in 2007 to $\$ 10235699$ in 2015. An increased tendency was evident for both outpatient and hospitalisation costs. However, because most patients with AGW are likely treated in outpatient clinics, the total cost of outpatient clinics in $2015 \$ 9$ 329287 was $10 \cdot 3$-fold that of hospitalisation $\$ 906$ 412. The costs per capita for hospitalisation increased considerably compared to those for outpatient clinics (Fig. 3 and Table 2).

\section{Direct medical cost}

The total direct medical cost increased about $2 \cdot 8$-fold during the last 9 years. Interestingly, the increase in

\footnotetext{
${ }^{1}$ Round the original estimates to 100000 South Korean Won $(\mathrm{KRW})$, then convert KRW into US\$ (1 US\$ = $1154 \mathrm{KRW})$.
}

payments by insurers and patients over the 9-year study period $(2 \cdot 9$-fold) was greater than that in prescribed pharmaceuticals (1.9-fold) (Fig. 3 and Table 2).

\section{Direct non-medical cost}

The direct non-medical cost for visiting outpatient clinics increased 2.4-fold from $\$ 74523$ in 2007 to $\$ 166377$ in 2015. The cost for hospitalisation in 2015 (\$11 265) was 2.2-fold that in 2007 (\$5199). The total direct non-medical cost increased from 2007 to 2014, and decreased in 2015 (Fig. 3 and Table 2).

\section{Indirect cost}

The total indirect cost increased 2.3-fold from $\$ 1117$ 850 in 2007 to $\$ 2608318$ in 2015 (Fig. 3 and Table 2).

\section{Co-morbidities of AGW}

Non-specific urethritis, chronic prostatitis and viral warts were the most prevalent co-morbidities in males with AGW. Sexually transmitted diseases (STDs) and inflammatory diseases (e.g. acute vaginitis, vulvar or vaginal candidiasis, and cervicitis) were the most frequent co-morbidities in females (Fig. 4).

\section{DISCUSSION}

We present interesting insight into the trends in annual AGW prevalence and the associated socio-economic costs nationwide. We hypothesised that the prevalence of AGW in females would fall after the introduction of HPV vaccination. Although HPV vaccines preventing AGW have been available since 2007 to both 

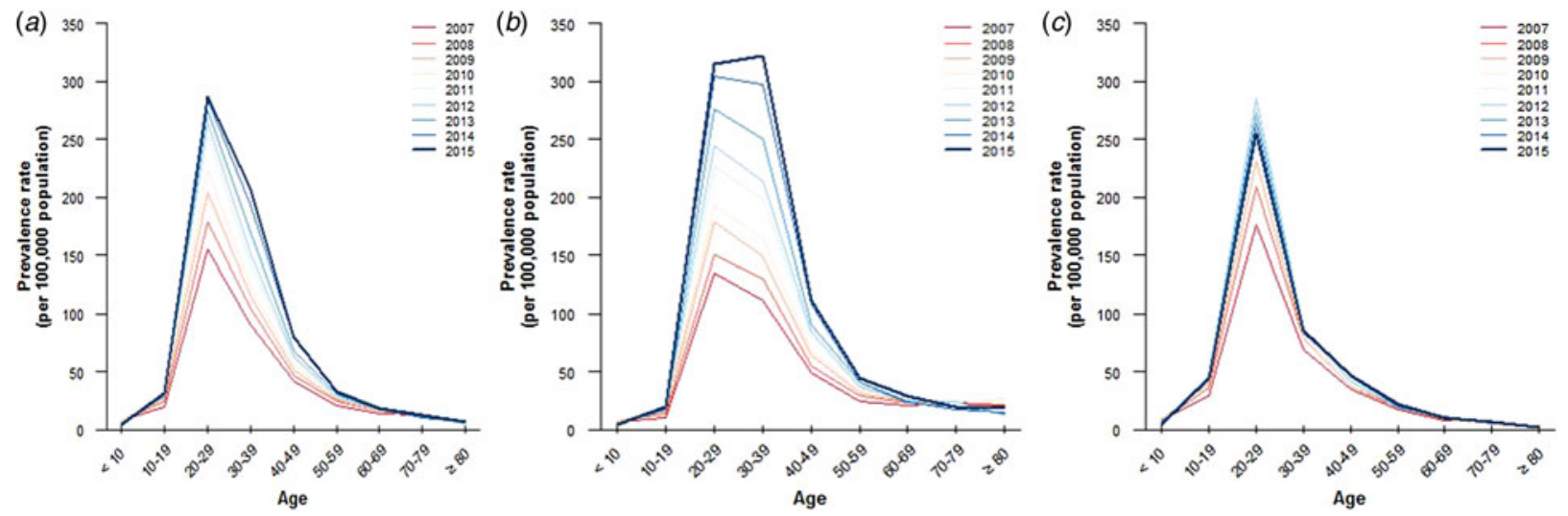

Fig. 2. Prevalence of anogenital warts according to sex and ages in Korea, 2007-2015. (a) Prevalence of anogenital warts in the whole population, $(b)$ in male population and $(c)$ in female population.
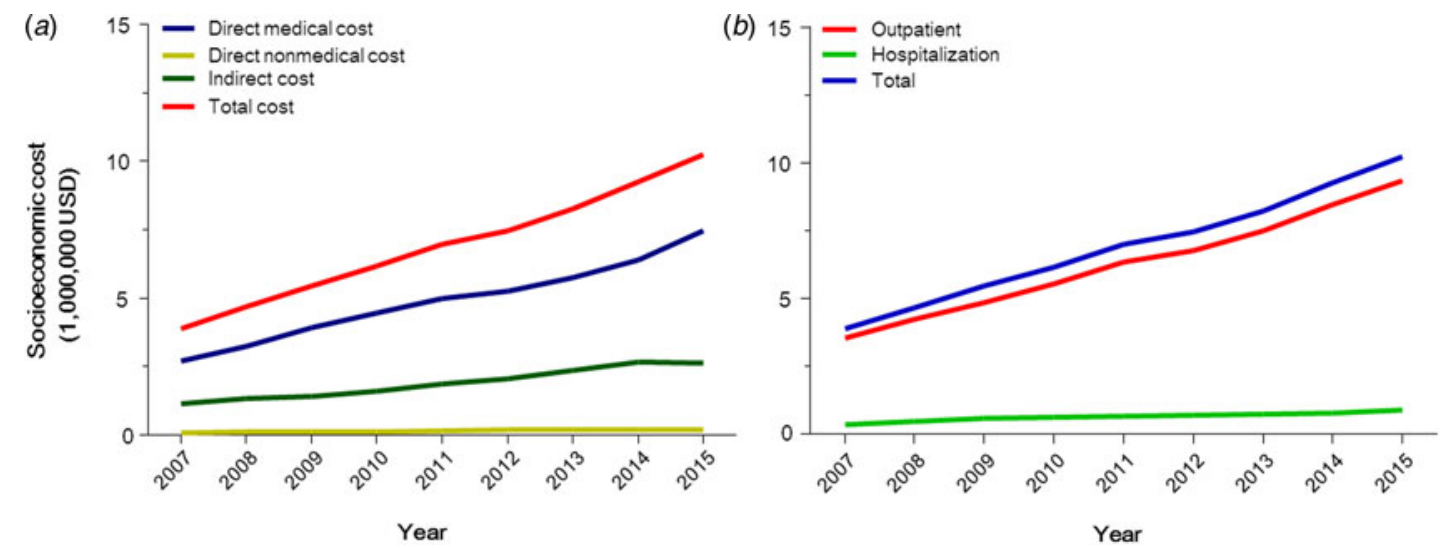

Fig. 3. Socio-economic cost of anogenital warts in Korea, 2007-2015. (a) Socio-economic cost in accordance with cost types and $(b)$ with service types.

sexes, most vaccinated subjects have been female. However, HPV vaccination became a component of the NIP only in 2016, and the number of subjects vaccinated is unknown. Therefore, the impact of vaccination on disease prevalence cannot be precisely estimated.

HPV vaccination effectively prevents AGW and cervical cancer in females. Among HPV-naïve females aged 16-26 years, HPV vaccination is almost $100 \%$ effective against AGW caused by low-risk HPV types, and $83 \%$ effective against AGW caused by any HPV type [30]. Vaccination protects against HPV infection for a minimum of 5 years with an efficacy $>90 \%$ [31]. A recent systematic review reported that HPV vaccination decreased the risk of AGW by $61 \%$ in girls aged $13-19$ years, and $68 \%$ in women aged 20-39 years [32].

HPV vaccination also protects males against AGW caused principally by non-oncogenic HPV types 6 and
11. The HPV vaccine prevents HPV 6/11/16/18 infection and anogenital disease in males aged 16-26 years; it is $90 \cdot 4 \%$ effective against external genital lesions caused by HPV $6,11,16$ or 18 , and $83.8 \%$ effective against external genital lesions caused by any HPV type [33].

Thus, it is likely that the decrease in AGW prevalence in females evident after 2012 is attributable to HPV vaccination. The AGW prevalences in 2007 were similar in both sexes, but this changed in later years. The male prevalence increased continuously, whereas the female prevalence decreased after 2012.

Notably, the AGW prevalence in females increased to 2012, but decreased thereafter (5 years after introduction of the HPV vaccine). The HPV (types 6/11/ 16/18) prevalence in the US declined by $56 \% 4$ years after HPV vaccine introduction, from $11.5 \%$ in $2003-2006$ to $5 \cdot 1 \%$ in $2007-2010$ (the post-vaccine period) [34]. In Australia, the AGW prevalence also decreased 4 years after the introduction of HPV 
Table 2. Socio-economic cost of anogenital warts in Korea, 2007-2015

\begin{tabular}{|c|c|c|c|c|c|c|c|c|}
\hline \multirow[b]{2}{*}{ Year } & \multirow[b]{2}{*}{ Category } & \multicolumn{3}{|c|}{ Direct medical cost } & \multirow{2}{*}{$\begin{array}{l}\text { Direct non- } \\
\text { medical cost } \\
\text { Traffic } \\
\text { expense* }\end{array}$} & \multirow{2}{*}{$\begin{array}{l}\text { Indirect cost } \\
\text { Lost } \\
\text { productivity* }\end{array}$} & \multicolumn{2}{|l|}{ Total cost } \\
\hline & & $\begin{array}{l}\text { Paid by insurer } \\
\text { and patients* }\end{array}$ & $\begin{array}{l}\text { Prescribed } \\
\text { pharmaceuticals* }\end{array}$ & $\begin{array}{l}\text { Total direct } \\
\text { medical cost* }\end{array}$ & & & $\begin{array}{l}\text { Total } \\
\text { cost* }\end{array}$ & $\begin{array}{l}\text { Per } \\
\text { capita* }\end{array}$ \\
\hline \multirow[t]{3}{*}{2007} & Outpatient & 2006065 & 430675 & 2436740 & 74523 & 1026863 & 3538126 & 134 \\
\hline & Hospitalisation & 266897 & - & 266897 & 5199 & 90987 & 363083 & 921 \\
\hline & Total & 2272962 & 430675 & 2703637 & 79722 & 1117850 & 3901209 & 146 \\
\hline \multirow[t]{3}{*}{2008} & Outpatient & 2449740 & 460138 & 2909878 & 100519 & 1207972 & 4218369 & 140 \\
\hline & Hospitalisation & 335355 & - & 335355 & 6932 & 115251 & 457538 & 1070 \\
\hline & Total & 2785095 & 460138 & 3245233 & 107452 & 1323223 & 4675908 & 153 \\
\hline \multirow[t]{3}{*}{2009} & Outpatient & 2925476 & 562391 & 3487867 & 110051 & 1277296 & 4875214 & 147 \\
\hline & Hospitalisation & 431542 & - & 431542 & 7798 & 135181 & 574521 & 1187 \\
\hline & Total & 3357018 & 562391 & 3919409 & 117849 & 1412477 & 5449735 & 163 \\
\hline \multirow[t]{3}{*}{2010} & Outpatient & 3334488 & 643847 & 3978335 & 124783 & 1441074 & 5544192 & 156 \\
\hline & Hospitalisation & 454939 & - & 454939 & 7798 & 141247 & 603984 & 1228 \\
\hline & Total & 3789427 & 643847 & 4433274 & 132581 & 1582321 & 6148176 & 171 \\
\hline \multirow[t]{3}{*}{2011} & Outpatient & 3792894 & 711438 & 4504332 & 145580 & 1684575 & 6334487 & 161 \\
\hline & Hospitalisation & 489601 & - & 489601 & 9532 & 156845 & 655978 & 1234 \\
\hline & Total & 4282495 & 711438 & 4993933 & 155112 & 1841420 & 6990465 & 176 \\
\hline \multirow[t]{3}{*}{2012} & Outpatient & 4088388 & 654246 & 4742634 & 163778 & 1873483 & 6779895 & 168 \\
\hline & Hospitalisation & 520797 & - & 520797 & 10398 & 165511 & 696706 & 1285 \\
\hline & Total & 4609185 & 654246 & 5263431 & 174176 & 2038994 & 7476601 & 182 \\
\hline \multirow[t]{3}{*}{2013} & Outpatient & 4538128 & 642980 & 5181108 & 170710 & 2155979 & 7507797 & 176 \\
\hline & Hospitalisation & 551126 & - & 551126 & 11265 & 181109 & 743500 & 1285 \\
\hline & Total & 5089254 & 642980 & 5732234 & 181975 & 2337088 & 8251297 & 191 \\
\hline \multirow[t]{3}{*}{2014} & Outpatient & 5100519 & 714038 & 5814557 & 176776 & 2459272 & 8450605 & 184 \\
\hline & Hospitalisation & 590987 & - & 590987 & 11265 & 194107 & 796359 & 1295 \\
\hline & Total & 5691506 & 714038 & 6405544 & 188041 & 2653379 & 9246964 & 199 \\
\hline \multirow[t]{3}{*}{2015} & Outpatient & 5927209 & 835355 & 6762564 & 166377 & 2400346 & 9329287 & 197 \\
\hline & Hospitalisation & 687175 & - & 687175 & 11265 & 207972 & 906412 & 1352 \\
\hline & Total & 6614384 & 835355 & 7449739 & 177642 & 2608318 & 10235699 & 213 \\
\hline
\end{tabular}

* Unit: US\$.

vaccination, from $18 \cdot 6 \%$ in females in the pre-vaccine era $(2004-\operatorname{mid} 2007)$ to $1.9 \%$ in the post-vaccine period (mid 2007-2011), and from $22.9 \%$ to $2 \cdot 9 \%$ in males [21]. If the decline in AGW in Korea is attributable to HPV vaccination, this should continue given that the NIP commenced in 2016. Data from the next few years should confirm this suggestion.

We found that the AGW prevalences in different age groups differed from those in Western counterparts. The AGW prevalence in Western countries is highest in those aged 20-29 years, followed by those aged 10-19 years [19]. However, we found higher overall AGW prevalences in older Korean patients. The prevalence was higher in males aged 20-49 years than in other age groups. The prevalences in those aged 20-29 and 30-39 years, and even 40-49 and 50-59 years, were higher than in those aged 1019 years. This is likely due to less sexual activity by Korean teenagers compared with their Western counterparts. For example, $41 \cdot 2 \%$ of high school students in the US have had sexual intercourse, compared with $10 \cdot 5 \%(7 \cdot 3 \%$ and $3 \cdot 2 \%$ in male and female adolescents, respectively) in Korea [35, 36]. The prevalence of AGW in males aged 20-29 years was higher than that in those aged 30-39 years to 2014 , but reversed thereafter. Interestingly, a sex difference in prevalence was apparent by age group. The prevalence of AGW in males peaked twice, in those aged 20-29 and 30-39 years, but only one peak was evident in females (aged 20-29 years). This may be attributable to differences in the sexual activities of young males and females. Thus, encouraging HPV vaccination of males may reduce the rapidly increasing AGW prevalence in Korea. In Australia, an HPV vaccination NIP significantly reduced the AGW prevalence by $81 \cdot 8 \%$ in males aged $<21$ years (thus from $12 \cdot 1 \%$ to $2 \cdot 2 \%$ ) and by $51 \cdot 1 \%$ (from $18 \cdot 2 \%$ to $8 \cdot 9 \%$ ) in males aged $21-30$ years [19]. 
(a) Male

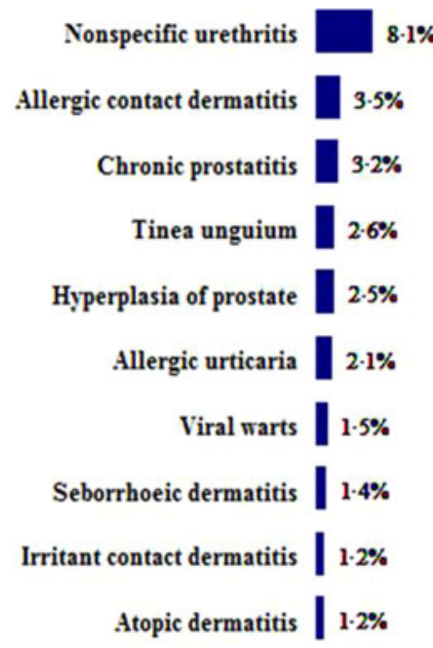

(b) Female

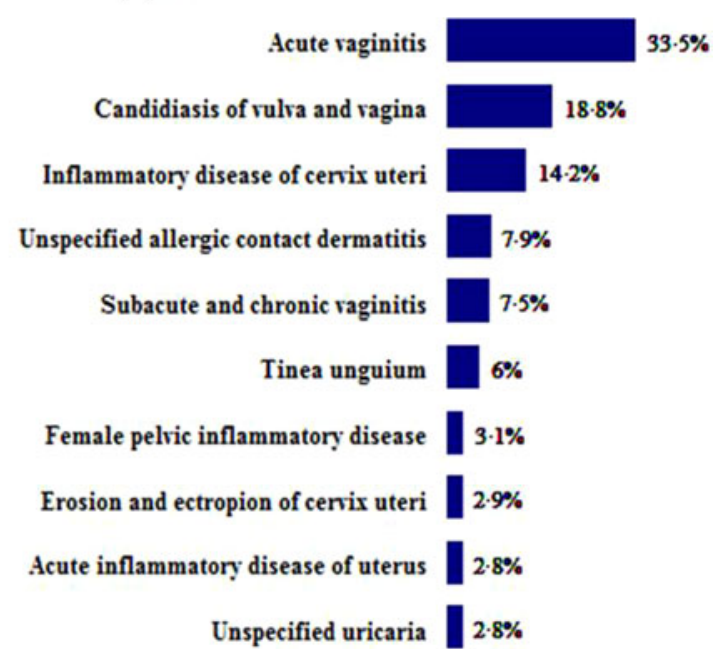

Fig. 4. Most frequent co-morbidities in male and female patients with anogenital warts.

We present reliable data on the socio-economic burdens imposed AGW, including direct medical, nonmedical and indirect costs. All of these costs increased $2 \cdot 2-2 \cdot 8$-fold during the 9 -year study period, probably because of the increase in overall AGW prevalence. The total costs of outpatient visits in 2015 also were $10 \cdot 3$-fold those of hospitalisation. This is because AGW are usually treated in outpatient clinics. However, one limitation of our study is that differences in disease severity and stage were not considered when we made these estimates. Furthermore, AGW are accompanied by co-morbidities such as STDs or inflammatory diseases of the anogenital area (e.g. urethritis and prostatitis in males, and candidiasis or other inflammatory diseases of the vulva and vagina in females). As such diseases are often STIs, patients with AGW are at a high risk of additional STIs. Physicians should screen AGW patients for STI co-morbidities.

This study was limited by the fact that only patients with the A63.0 code were included in the calculation of prevalence and socio-economic burden. The inclusion of all cases with the A63, A63.0 and A63.8 diagnostic codes, together with recurrent cases, would increase the number of cases, prevalence and economic burden of AGW. This study did not examine the epidemiological differences in AGW according to HPV genotype despite the fact that the prevalence and socio-economic burden differ among the HPV genotypes. Furthermore, the exact site of the infection or whether it was multifocal was not clear because this study examined claims data for patients with 'AGW' combining all localisations into one group of 'anogenital sites'. Therefore, the exact localisation of the warts was not indicated. Further studies of the characteristics of multiple genital infections can provide information on the dynamics of patients with anogenital HPV infection.

In conclusion, HPV infection and related diseases such as AGW are of clinical concern in Korea, attributable to their high prevalence, adverse economic effects and co-morbidities. We found that the overall AGW prevalence and socio-economic burden imposed thereby increased over the 9 years of our study. However, the prevalence of AGW differed significantly by sex. Whereas the male prevalence increased continuously over time, the female prevalence fell after 2012. The prevalence of, and economic burden imposed by, HPV-related diseases will continue to increase in males if no action is taken. Therefore, increased HPV surveillance in males, and implementation of malespecific preventative measures, should be considered.

\section{SUPPLEMENTARY MATERIAL}

The supplementary material for this article can be found at https://doi.org/10.1017/S0950268817002813

\section{ACKNOWLEDGEMENTS}

This work was supported by the Soonchunhyang University. The authors acknowledge useful advice provided by Merck Sharp \& Dohme Corp. The opinions expressed in this paper are those of the authors and do not necessarily represent those of Merck Sharp \& Dohme Corp. This work was supported in part by 
a research grant from Investigator-Initiated Studies Programme of Merck Sharp \& Dohme Corp. The opinions expressed in this paper are those of the authors and do not necessarily represent those of Merck Sharp \& Dohme Corp.

\section{CONFLICTS OF INTEREST}

None.

\section{ETHICAL STANDARDS}

This study got an ethical approval from the Institutional Review Board (IRB) of Soonchunhyang University Bucheon Hospital (IRB no. SCHBC 2015-03-008). The authors assert that all procedures contributing to this work comply with the ethical standards of the relevant national and institutional guides regarding data collection and analysis.

\section{REFERENCES}

1. Patel H, et al. Systematic review of the incidence and prevalence of genital warts. BioMed Central Infectious Diseases 2012; 13: 39-39.

2. Winer RL. Genital human papillomavirus infection: incidence and risk factors in a cohort of female university students. American Journal of Epidemiology 2003; 157: 218-226.

3. Castle PE, Maza M. Prophylactic HPV vaccination: past, present, and future. Epidemiology and Infection 2016; 144: 449-468.

4. International Human Papillomavirus Reference Center. Human papillomavirus reference clones. (http://www. hpvcenter.se/html/refclones.html). Accessed 24 October 2017.

5. Insinga RP, Dasbach EJ, Myers ER. The health and economic burden of genital warts in a set of private health plans in the United States. Clinical Infectious Diseases 2003; 36: 1397-1403.

6. Capra G, et al. Analysis of persistence of human papillomavirus infection in men evaluated by sampling multiple genital sites. European Review for Medical and Pharmacological Sciences 2015; 19: 4153-4163.

7. Pellati D, et al. Genital tract infections and infertility. European Journal of Obstetrics \& Gynecology and Reproductive Biology 2008; 140: 3-11.

8. Patel RV, Yanofsky VR, Goldenberg G. Genital warts: a comprehensive review. Journal of Clinical and Aesthetic Dermatology 2012; 5: 25-36.

9. Markowitz LE, et al. Human papillomavirus vaccination: recommendations of the Advisory Committee on Immunization Practices (ACIP). MMWR: Recommendations and Reports 2014; 63: 1-30.

10. Satterwhite CL, et al. Sexually transmitted infections among US women and men: prevalence and incidence estimates, 2008. Sexually Transmitted Diseases 2013; 40: $187-193$.

11. Shin H-R, et al. Prevalence and determinants of genital infection with papillomavirus, in female and male university students in Busan, South Korea. Journal of Infectious Diseases 2004; 190: 468-476.

12. Park SJ, et al. Prevalence and determinants of high-risk human papillomavirus infection in male genital warts. Korean Journal of Urology 2014; 55: 207-212.

13. Centers for Disease Control Prevention. Recommendations on the use of quadrivalent human papillomavirus vaccine in males - advisory committee on immunization practices (ACIP), 2011. MMWR Morbidity and Mortality Weekly Report 2011; 60: 1705.

14. Kim MK, No JH, Song YS. Human papillomavirus vaccine. Journal of the Korean Medical Association 2009; 52: $1180-1186$

15. Anon. Human papillomavirus vaccines. WHO position paper. Weekly Epidemiological Record 2009; 84: 118-131.

16. Anon. Human papillomavirus vaccines: WHO position paper, October 2014-recommendations. Vaccine 2015; 33: 4383-4384.

17. Capra G, et al. Potential impact of a nonavalent HPV vaccine on HPV related low-and high-grade cervical intraepithelial lesions: a referral hospital-based study in Sicily. Human Vaccines \& Immunotherapeutics 2017; 13: $1839-1843$

18. Li N, et al. Human papillomavirus type distribution in 30,848 invasive cervical cancers worldwide: variation by geographical region, histological type and year of publication. International Journal of Cancer 2011; 128: 927-935.

19. Ali H, et al. Genital warts in young Australians five years into national human papillomavirus vaccination programme: national surveillance data. British Medical Journal 2013; 346: f2032.

20. Hartwig S, et al. Estimation of the epidemiological burden of human papillomavirus-related cancers and nonmalignant diseases in men in Europe: a review. $B M C$ Cancer 2012; 12: 30.

21. Read TR, et al. The near disappearance of genital warts in young women 4 years after commencing a national human papillomavirus (HPV) vaccination programme. Sexually Transmitted Infections 2011; 87: 544-547.

22. World Health Organization. International Statistical Classification of Diseases and Related Health Problems. 10th edn. Geneva, CH: World Health Organization, 1989.

23. Korean Statistical Information Service. Korea national statistical office. (http://kosis.kr/statisticsList/statisticsList_01 List.jsp?vwcd=MT_ZTITLE\&parentId=A) 2017.

24. Jung YH, Ko S. The socioeconomic cost of diseases in Korea. Journal of Preventive Medicine and Public Health Yebang Uihakhoe Chi 2006; 39: 499-504.

25. Park CS, et al. Cost-of-illness study of asthma in Korea: estimated from the Korea National Health insurance claims database. Journal of Preventive Medicine and Public Health Yebang Uihakhoe Chi 2006; 39: 397-403.

26. National Health Insurance Corporation. Survey of Medical Charges Paid by Patients with Health Insurance in 2010. Seoul: National Health Insurance Corporation, 2010. 
27. Korea Institute for Health and Social Affairs (KIHASA). The basic analysis report of Korean health panel survey in 2008. (https://www.kihasa.re.kr/web/activity/research/view. do? menuId $=39 \&$ tid $=72 \&$ bid $=93 \&$ division $=003 \&$ ano $=545$ ) 2017.

28. Drummond MF, et al. Methods for the Economic Evaluation of Health Care Programmes. 3rd edn. Oxford, UK: Oxford University Press, 2005.

29. No IC, Suh MH, Kim YL. Socioeconomic Costs of Alcohol and Policy Issues. Seoul: Korea Institute of Health and Social Affairs, 1997.

30. Future I/II Study Group, et al. Four year efficacy of prophylactic human papillomavirus quadrivalent vaccine against low grade cervical, vulvar, and vaginal intraepithelial neoplasia and anogenital warts: randomised controlled trial. BMJ: British Medical Journal 2010; 341: c3493.

31. Garland SM, et al. Quadrivalent vaccine against human papillomavirus to prevent anogenital diseases. New England Journal of Medicine 2007; 356: 1928-1943.
32. Drolet M, et al. Population-level impact and herd effects following human papillomavirus vaccination programmes: a systematic review and meta-analysis. Lancet Infectious Diseases 2015; 15: 565-580.

33. Giuliano AR, et al. Efficacy of quadrivalent HPV vaccine against HPV infection and disease in males. New England Journal of Medicine 2011; 364: 401-411.

34. Markowitz LE, et al. Reduction in human papillomavirus (HPV) prevalence among young women following HPV vaccine introduction in the United States, National Health and Nutrition Examination Surveys, 2003-2010. Journal of Infectious Diseases 2013; 208: 385-393.

35. Kann L, et al. Youth risk behavior surveillance - United States, 2015. MMWR Surveillance Summaries 2016; 65: $1-174$.

36. Seoul Mental Health Statistics. A study based on the 10th (2014) adolescent health behavior online survey. (https://www.seoulmentalhealth.kr/dataroom/data.jsp? cmd=view\&test_num=63) 2017. 